Volume 128, Number 10, Pages 2869-2874

S 0002-9939(00)05476-9

Article electronically published on April 7, 2000

\title{
RELATIONS BETWEEN CUSP FORMS ON CONGRUENCE AND NONCONGRUENCE GROUPS
}

\author{
GABRIEL BERGER
}

(Communicated by David E. Rohrlich)

\begin{abstract}
We construct a quadratic relation between cusp forms of weight two on four genus 1 subgroups of $S L_{2}(\mathbb{Z})$. Two of the subgroups are congruence and two are noncongruence. We then generalize this to subgroups of $\Gamma(N)$ of index 2 .
\end{abstract}

Let $\Delta$ be a finite-index subgroup of $S L_{2}(\mathbb{Z})$, and let $X_{\Delta}$ be the associated Riemann surface $\mathfrak{h}^{*} / \Delta$. If $K$ is a number field and $C / K$ is a projective nonsingular curve, we say that $C / K$ is a model for $X_{\Delta}$ if there exists a morphism $\phi: C \rightarrow \mathbb{P}^{1}$ defined over $K$ and a complex analytic isomorphism $p: X_{\Delta} \rightarrow C(\mathbb{C})$ such that $p(\infty)$ is rational over $K$ and

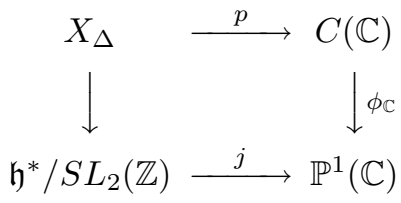

commutes. (Here, the left hand arrow is the natural projection map.) It is known that for any $\Delta$ one can find a $C / K$ as above, and, in fact, Belyi's theorem $[\mathbb{B}$ tells us that the converse is true as well. In light of this, one may wish to study the usual arithmetic objects associated with congruence groups (i.e., Hecke operators, Fourier coefficients, and so forth) for arbitrary subgroups of finite index in $S L_{2}(\mathbb{Z})$. While it is true that on a general noncongruence subgroup, Hecke operators and Fourier coefficients are not related, there are nevertheless results concerning both. (See $[\mathrm{Be}]$ for Hecke operators on noncongruence subgroups and $[\mathrm{S}]$ for Fourier coefficients. From $[\mathrm{Be}]$, we see that Hecke operators on noncongruence subgroups give essentially no new information, but the Atkin-Swinnerton-Dyer congruences of $[\underline{S}$ are highly nontrivial.) What has not been previously studied, however, is the relationship between cusp forms on related congruence and noncongruence groups. Since cusp forms on congruence groups are to some extent understood, any explicit information we obtain may be viewed as shedding light on the noncongruence forms. In particular, an actual polynomial relationship between forms would lead to an infinite number of relations between Fourier coefficients. One may view this as a

Received by the editors November 16, 1998.

1991 Mathematics Subject Classification. Primary 11F11; Secondary 11F30.

Key words and phrases. Noncongruence subgroup, cusp form.

The author was supported in part by JSPS grant P94015 and NSA grant 032596.

(C)2000 American Mathematical Society 
small step in the direction of understanding the arithmetic significance of Fourier coefficients of noncongruence cusp forms.

In this note, we study relations as above between cusp forms on varying finite index subgroups of $S L_{2}(\mathbb{Z})$. The subgroups are all of index 2 in a congruence group and are obtained through pure group theory. In Theorem 1, we exhibit four explicit genus 1 subgroups of $\Gamma_{0}(16)$ and an explicit quadratic relation between the unique normalized cusp forms on each group. We then generalize this. In Theorem 2 we show that a quadratic relation exists between cusp forms on subgroups of index 2 in $\Gamma(N)$ for all sufficiently large $N$. In particular, the cusp forms may be taken to all belong to different groups.

Theorem 1. There exist four genus 1 subgroups $\Delta_{1}, \Delta_{2}, \Delta_{3}, \Delta_{4}$ of finite index in $S L_{2}(\mathbb{Z})$ with the following properties:

1) $\Delta_{1}$ and $\Delta_{2}$ are congruence.

2) $\Delta_{3}$ and $\Delta_{4}$ are noncongruence.

3) $X_{\Delta_{1}}$ has a model of conductor 32 .

4) $X_{\Delta_{2}}$ has a model of conductor 64 .

5) $X_{\Delta_{3}}$ has a model of conductor 128.

6) $X_{\Delta_{4}}$ has a model of conductor 128 .

7) Denote by $S_{2}\left(\Delta_{i}\right)$ the space of cusp forms of weight 2 on $\Delta_{i}$, and let $f_{i} \in$ $S_{2}\left(\Delta_{i}\right)$ be the unique such cusp form with first Fourier coefficient equal to 1. Then

$$
4 f_{1}^{2}-f_{2}^{2}=2 f_{3}^{2}-f_{4}^{2}
$$

Proof. We begin by defining

$$
\mu(z)=\frac{1}{16 q} \prod_{n=1}^{\infty}\left(\frac{1+q^{2 n-1}}{1+q^{2 n}}\right)^{8},
$$

for $q=e^{2 \pi i z}$. Then it is known (see $[\mathrm{F}]$ ) that $\mu(z)$ is a Hauptmodul for $\Gamma_{0}(4)$ (i.e., it generates the function field of $\left.X_{0}(4)\right)$, and we note that $\Gamma_{0}(4)$ has three inequivalent cusps, representatives of which may be taken to be $0,1 / 2$, and $\infty$. It is easily verified that $\mu(0)=1, \mu(1 / 2)=0$, and $\mu(\infty)=\infty$.

Now, $X_{0}(16)$ also has genus 0 , and thus has a Hauptmodul $x$. We specify $x$ uniquely by declaring that

$$
x(0)=0, x\left(\frac{1}{2}\right)=\infty, \text { and } x(\infty)=-1 .
$$

In addition $\Gamma_{0}(16)$ is known to have six inequivalent cusps, representatives of which may be taken to be $0,1 / 2, \pm 1 / 4,1 / 8$, and $\infty$. In the cover $X_{0}(16) \rightarrow X_{0}(4)$, the first 2 points have ramification index 4 (over 0 and $1 / 2$, respectively), and the last 4 (all over $\infty$ ) have ramification index 1 . We may thus define a map $\pi: \mathbb{P}^{1} \rightarrow \mathbb{P}^{1}$ such that

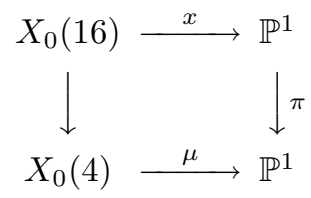

is commutative; the considerations above tell us that $\pi(t)=1 /\left(1-t^{4}\right)$. Thus $\mu=1 /\left(1-x^{4}\right)$. Using this, one sees (see also $[\mathbf{F}]$ ) that $x(\{1 / 4,3 / 4\})=\{ \pm i\}$ and 
$x(1 / 8)=1$. The Fourier expansion of $x$ is

$$
x=-1+4 e^{2 \pi i z}-8^{4 \pi i z}+\ldots
$$

For each cusp $t$ as above of $\Gamma_{0}(16)$, we consider a generator $\gamma_{t}$ of $\Gamma_{0}(16)_{t}$, the stabilizer of $t$ in $\Gamma_{0}(16)$. Then $\Gamma_{0}(16)$ is generated by the $\gamma_{t}$, and the only relation between these generators is that their product in some order (possibly replacing $\gamma_{t}$ by $\gamma_{t}^{-1}$ ) is 1 . In particular, we may define homomorphisms

$$
\psi: \Gamma_{0}(16) \rightarrow\{ \pm 1\}
$$

by sending, say, two generators to 1 and the remaining 4 to -1 . The kernel $\Delta$ of this map will have index 2 in $\Gamma_{0}(16)$, and hence the canonical map $X_{\Delta} \rightarrow X_{0}(16)$ will have degree 2 and be ramified over 4 points. The Hurwitz formula then tells us that $X_{\Delta}$ will have genus 1 . We will choose 4 such homomorphisms $\psi_{1}, \ldots, \psi_{4}$, and these will give us the subgroups $\Delta_{1}, \ldots, \Delta_{4}$ of the theorem.

So set

$$
\begin{gathered}
\gamma_{0}=\left(\begin{array}{cc}
1 & 0 \\
16 & 1
\end{array}\right), \gamma_{\frac{1}{2}}=\left(\begin{array}{cc}
-7 & 4 \\
-16 & 9
\end{array}\right), \gamma_{\frac{1}{4}}=\left(\begin{array}{cc}
-3 & 1 \\
-16 & 5
\end{array}\right), \\
\gamma_{-\frac{1}{4}}=\left(\begin{array}{cc}
-3 & -1 \\
16 & 5
\end{array}\right), \gamma_{\frac{1}{8}}=\left(\begin{array}{cc}
-7 & 1 \\
-64 & 9
\end{array}\right), \text { and } \gamma_{\infty}=\left(\begin{array}{ll}
1 & 1 \\
0 & 1
\end{array}\right) .
\end{gathered}
$$

To define $\psi_{i}$ we need only specify which 2 generators are sent to 1 ; the other 4 will be sent to -1 . Our maps are as follows:

$$
\begin{aligned}
& \psi_{1}: \gamma_{\frac{1}{8}}, \gamma_{\infty} \mapsto 1, \\
& \psi_{2}: \gamma_{\frac{1}{4}}, \gamma_{-\frac{1}{4}} \mapsto 1, \\
& \psi_{3}: \gamma_{0}, \gamma_{\infty} \mapsto 1, \\
& \psi_{4}: \gamma_{0}, \gamma_{\frac{1}{8}} \mapsto 1
\end{aligned}
$$

Lemma 1. 1) $\Delta_{1}=\Gamma_{0}(32)$.

2) $\Delta_{2} \subset \Gamma_{0}(32) \cap \Gamma^{0}(2)$ as an index 2 subgroup.

3) $\Delta_{3}$ and $\Delta_{4}$ are noncongruence.

Proof. 1) and 2) One may explicitly write down generators for $\Gamma_{0}(32)$ and $\Gamma_{0}(32) \cap$ $\Gamma^{0}(2)$ in terms of generators for $\Gamma_{0}(16)$ and verify the claim generator by generator.

3) Note that $\Delta_{3}$ has level (= least common multiple of the cusp widths; see [Be]) equal to 16 . Thus if $\Delta_{3}$ were congruence, it would have to contain $\Gamma(16)$. Suppose this is the case. Choose $\beta \in \Gamma_{0}(16)$ such that $\beta \equiv\left(\begin{array}{ll}5 & -2 \\ 0 & -3\end{array}\right) \bmod 16$. Since $\left[\Gamma_{0}(16): \Delta_{3}\right]=2, \beta^{2} \in \Delta_{3}$. Now $\gamma_{\frac{1}{2}} \notin \Delta_{3}$, but

$$
\gamma_{\frac{1}{2}} \cdot \beta^{2} \equiv\left(\begin{array}{cc}
-7 & 4 \\
0 & 9
\end{array}\right)\left(\begin{array}{cc}
9 & -4 \\
0 & 9
\end{array}\right) \equiv\left(\begin{array}{cc}
1 & 0 \\
0 & 1
\end{array}\right) \in \Gamma_{0}(16)
$$

Thus $\gamma_{\frac{1}{2}} \beta^{2} \in \Delta_{3}$, a contradiction. The same proof carries over word for word for $\Delta_{4}$. (Note that we could also have calculated Fourier expansions of normalized cusp forms and shown, say, that their coefficients are not algebraic integers.) 
Since the $X_{\Delta_{i}}$ all have genus 1 , their function fields are of the form $\mathbb{C}(x, y)$, where $y^{2}=f_{i}(x)$ is a cubic polynomial in $x$.

In the case of $\Delta_{3}$, we see that the map $X_{\Delta_{3}} \rightarrow X_{0}(16)$ ramifies over $1 / 2, \pm 1 / 4$, $1 / 8$, and hence if we fill in the right hand arrow below to make a commutative diagram

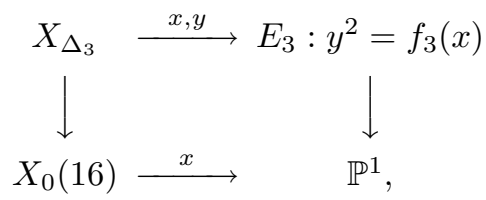

we see that $E_{3}$ ramifies over $x(\{1 / 2, \pm 1 / 4,1 / 8\})=\{\infty, \pm i, 1\}$. Thus, we obtain the affine equation $y^{2}=-\left(x^{2}+1\right)(x-1)$, an elliptic curve of conductor 128, which is clearly a model over $\mathbb{Q}$ for $X_{\Delta_{3}}$. (Note that the minus sign comes from the necessity for the image of $\infty$ to be rational, since $\left.\left(x^{2}+1\right)(x-1)=-4+24 e^{2 \pi i z} \ldots\right)$ Similarly, for $X_{\Delta_{4}}$ we obtain the equation $y^{2}=\left(x^{2}+1\right)(x+1) / 2$ of conductor 128 . For $X_{\Delta_{1}}$, we obtain the equation $y^{2}=-\left(x^{3}+x\right) / 2$ of conductor 32 , and for $X_{\Delta_{2}}$, the equation $y^{2}=\left(x^{3}-x\right) / 2$ of conductor 64 .

Conclusion of the Proof. Let $q=e^{\pi i z}$. Then for each group $\Delta_{i}$,

$$
\frac{q \cdot \frac{d x}{d q}}{y}
$$

is a cusp form of weight 2 on $\Delta_{i}$ for the appropriate $y$ as above. Note that in fact

$$
\begin{gathered}
\frac{1}{8} \cdot \frac{q \cdot \frac{d x}{d q}}{\sqrt{-\frac{x^{3}+x}{2}}}=q^{2}-2 q^{10}-3 q^{1} 8 \cdots=f_{1}, \\
\frac{1}{4} \cdot \frac{q \cdot \frac{d x}{d q}}{\sqrt{\frac{x^{3}-x}{2}}}=q+2 q^{5}-3 q^{9} \cdots=f_{2}, \\
\frac{1}{4} \cdot \frac{q \cdot \frac{d x}{d q}}{\sqrt{-\left(x^{2}+1\right)(x+1)}}=q^{2}-q^{4}-q^{6} / 2 \cdots=f_{3}, \\
\frac{1}{4} \cdot \frac{q \cdot \frac{d x}{d q}}{\sqrt{\frac{\left(x^{2}+1\right)(x+1)}{2}}}=q-q^{3}-q^{5} / 2 \cdots=f_{4} .
\end{gathered}
$$

Thus

$$
-4 f_{1}^{2}+f_{2}^{2}+2 f_{3}^{2}-f_{4}^{2}
$$

is equal to

$$
\frac{\left(q \cdot \frac{d x}{d q}\right)^{2}}{8} \cdot\left(\frac{1}{x^{3}+x}+\frac{1}{x^{3}-x}-\frac{1}{\left(x^{2}+1\right)(x-1)}-\frac{1}{\left(x^{2}+1\right)(x+1)}\right) .
$$

But this last expression is identically zero. This completes the proof of the theorem.

The basic principle used in the above is the following:

Proposition 1. Suppose $\Delta_{1}, \ldots, \Delta_{s}$ are distinct subgroups of index 2 in some subgroup $\Gamma$ of finite index in $S L_{2}(\mathbb{Z})$. For each $i, 1 \leq i \leq s$, let $S_{k}^{\text {prim }}\left(\Delta_{i}\right)$, the space 
of primitive cusp forms, denote the kernel of the trace map $\operatorname{Tr}_{i}: S_{k}\left(\Delta_{i}\right) \rightarrow S_{k}(\Gamma)$. For each $i$, choose a basis $\left\{f_{i, j}\right\}$ of a subspace $V_{i}$ of $S_{k}^{\text {prim }}\left(\Delta_{i}\right)$. Suppose that

$$
\sum_{i=1}^{s} \operatorname{dim} V_{i}>\operatorname{dim} S_{2 k}(\Gamma)
$$

Then the cusp forms $\left\{f_{i, j}\right\}$ satisfy a nontrivial quadratic relation:

$$
\sum_{i, j} a_{i, j} f_{i, j}^{2}=0
$$

for some complex numbers $a_{i, j}$.

Proof. For any $i, 1 \leq i \leq s$, suppose $\gamma_{i} \in \Gamma-\Delta_{i}$. Then for $f \in S_{k}\left(\Delta_{i}\right), \operatorname{Tr}_{i}(f)=$ $f+\left.f\right|_{\gamma_{i}}$. If $f \in S_{k}^{\text {prim }}\left(\Delta_{i}\right)$, then $f+\left.f\right|_{\gamma_{i}}=0$, so $f \mid \gamma_{i}=-f$. Thus $f^{2} \mid \gamma_{i}=f^{2}$, or $f^{2}$ is invariant under $\gamma$. But $f$ is invariant under $\Delta_{i}$ as well, so it is invariant under $\Gamma$. Thus $f^{2} \in S_{2 k}(\Gamma)$. Note also that if $(i, j) \neq(k, l)$, then $f_{i, j} \neq \pm f_{k, l}$, so $f_{i, j}^{2} \neq f_{k, l}^{2}$. The cusp forms $\left\{f_{i, j}^{2}\right\}$ then form $\sum_{i=1}^{s} \operatorname{dim} V_{i}$ distinct nonzero elements in a space of smaller dimension $\operatorname{dim} S_{2 k}(\Gamma)$. They must therefore satisfy a nontrivial linear relation.

To illustrate the above proposition, we consider the case $\Gamma=\Gamma(N)$ and $k=2$.

Lemma 2. If $\Delta$ has index 2 in $\Gamma(N)$ and is congruence, the level of $\Delta$ must be $N$ or $2 N$.

Proof. Since all cusps in $\Gamma(N)$ have width $N$ and $\Delta$ has index 2 in $\Gamma(N)$, all cusps of $\Delta$ will have width $N$ or $2 N$. Since the level of a finite index subgroup of $S L_{2}(\mathbb{Z})$ is the least common multiple of its cusp widths, the result follows.

It is interesting to note that the number of such $\Delta$ that are congruence is quite small:

Lemma 3. The number of $\Delta$ of index 2 in $\Gamma(N)$ that are congruence is 1 if $N$ is odd and 7 if $N$ is even.

Proof. By Lemma 2, any such $\Delta$ contains $\Gamma(2 N)$. Thus, we must count the number of subgroups of index 2 in the finite group $\Gamma(N) / \Gamma(2 N)$. But if $N$ is odd, then

$$
\Gamma(N) / \Gamma(2 N) \cong \Gamma(1) / \Gamma(2) \cong S_{3},
$$

and if $N$ is even, then

$$
\Gamma(N) / \Gamma(2 N) \cong \Gamma(2) / \Gamma(4) \cong(\mathbb{Z} / 2 \mathbb{Z})^{3} .
$$

But clearly, $S_{3}$ has only one subgroup of order 3 , whereas $(\mathbb{Z} / 2 \mathbb{Z})^{3}$ has 7 subgroups of order 4 .

Lemma 4 ( $\underline{\underline{S h}})$. Let

$$
\mu_{N}=N^{3} / 2 \prod_{p \mid N}\left(1-p^{-2}\right), h_{N}=\mu_{N} / N, \text { and } g_{N}=1+\mu_{N}(N-6) / 12 N .
$$

Then $\mu_{N}=\# P S L_{2}(\mathbb{Z} / N)=\frac{1}{2} \# S L_{2}(\mathbb{Z} / N), g_{N}$ is the genus of $\mathfrak{h}^{*} / \Gamma(N)$, and $h_{N}$ is the number of cusps of $\mathfrak{h}^{*} / \Gamma(N)$. Furthermore, $\Gamma(N)$ has no elliptic elements and is thus the fundamental group of the open surface $\mathfrak{h} / \Gamma(N)$.

Lemma 5. The number of subgroups of $\Gamma(N)$ of index 2 is $2^{r_{N}}-1$, where $r_{N}=$ $2 g_{N}+h_{N}-1$. 
Proof. Since $\Gamma(N)$ is the fundamental group of $\mathfrak{h} / \Gamma(N)$, and $\mathfrak{h} / \Gamma(N)$ is a Riemann surface of genus $g_{N}$ with $h_{N}$ punctures, topology tells us that $\Gamma(N)$ is free on $r_{N}=2 g_{N}+h_{N}-1$ generators. But then subgroups of index 2 in $\Gamma(N)$ correspond to surjective homomorphisms $\Gamma(N) \rightarrow \mathbb{Z} / 2 \mathbb{Z}$, which are determined by choosing the image of each generator, with the requirement that not every generator maps to 0 .

Lemma 6. $\operatorname{dim} S_{4}(\Gamma(N))=3\left(g_{N}-1\right)+h_{N}$. If $\Delta$ has index 2 in $\Gamma(N)$, then $\operatorname{dim} S_{2}(\Delta)=2 g_{N}-1+a_{N} / 2$, where $a_{N}$ is the number of ramified points in the cover $\mathfrak{h}^{*} / \Delta \rightarrow \mathfrak{h}^{*} / \Gamma(N)$. Furthermore, $\operatorname{dim} S_{2}^{\text {prim }}(\Delta)=g_{N}-1+a_{N} / 2$.

Proof. The first assertion follows from [Sh p. 23]. The second follows from the Riemann-Hurwitz formula: if $g$ is the genus of $\mathfrak{h}^{*} / \Delta$, then $\operatorname{dim}\left(S_{2}(\Delta)\right)=g$, and

$$
2 g-2=2\left(2 g_{N}-2\right)+a_{N} \text {, or } g=2 g_{N}-1+a_{N} / 2 \text {. }
$$

The last statement follows from the observation that $\operatorname{dim} S_{2}^{\text {prim }}(\Delta)=\operatorname{dim} S_{2}(\Delta)-$ $\operatorname{dim} S_{2}(\Gamma(N))$.

Using this, we can easily satisfy the conditions of Proposition 1,

Theorem 2. For sufficiently large $N$, there exists a nontrivial quadratic relation between cusp forms on subgroups of index 2 in $\Gamma(N)$. In particular, we may choose the cusp forms to all belong to different groups.

Proof. Note that since

$$
g_{N}>0 \text { and } 2^{r_{N}}-1=2^{2 g_{N}+h_{N}-1}-1>3\left(g_{N}-1\right)+h_{N}
$$

for suffiently large $N$, the number of subgroups of index 2 in $\Gamma(N)$ is greater than $\operatorname{dim} S_{4}(\Gamma(N))$ for such $N$. Hence, choosing a nonzero subspace $V \subset S_{k}(\Delta)$ for each $\Delta$ of index 2 in $\Gamma(N)$, we see that the conditions of the proposition are satisfied. Thus the cusp forms on all the subspaces $V$ satisfy a quadratic relation. In particular, we may choose each $V$ to have dimension 1, which means that each cusp form would belong to a different group.

\section{REFERENCES}

[B] Belyi, G.V. On the Galois Extensions of the Maximal Cyclotomic Field 1979, Izv. Akad. Nauk. SSSR, vol 43, pp. 267-276. MR 80f:12008

[Be] Berger, G. Hecke Operators on Noncongruence Subgroups 1994, C.R. Acad Sci, Paris, t 319, Series 1, pp. 915-919. MR 95k:11063

[F] Fricke, R. Lehrbuch der Algebra vol 2, 1926,Vieweg and Son, Braunschweig.

[S] Scholl, A.J. Modular Forms and de Rham Cohomology; Atkin-Swinnerton-Dyer congruences Invent. Math., vol 79, pp. 49-77. MR 86j:11045

[Sh] Shimura, G. Introduction to the Arithmetic Theory of Automorphic Functions Iwanami Shoten, 1971. MR 47:3318

Department of Mathematics, Boston College, Chestnut Hill, Massachusetts 02467 Current address: Alphatech, Inc., 50 Mall Rd., Burlington, Massachusetts 01803

E-mail address: gberger@channel1.com 